\title{
Michel Henry lecteur de Claude Tresmontant : création, révélation, écritures
}

\author{
Joaquim Hernandez-Dispaux \\ (Université Catholique de Louvain, \\ Fonds Michel Henry)
}

Cet article est le fruit d'un contact avec les notes préparatoires qui ont servi à la rédaction de la trilogie ${ }^{1}$. Celles-ci constituent le moment final de l'œuvre de Michel Henry qu'il consacre à une philosophie du christianisme. Comme souvent chez cet auteur, le manuscrit s'avère plus bavard que le texte publié. Parmi les sources qui y sont mobilisées, certaines seraient palpables par le biais ses nombreuses publications. Tel est le cas des œuvres abondamment citées dans le corpus henryen - on pense ici, par exemple, à celles de Hegel et Heidegger. D'autres références sont, en revanche, plus discrètes voire imperceptibles sans un recours aux « archives ». Leur influence n'en reste pas moins décisive : non seulement pour acquérir une meilleure intelligence des rapports qu'entretient Michel Henry au christianisme mais également pour relire, à l'aune de la phénoménologie de la vie, certains auteurs dont les thèses acquirent alors un sens inattendu. Claude Tresmontant (1925-1997) fait partie de ces auteurs rendus quasiment anonymes ${ }^{2}$ par leur assimilation dans la phénomé-

\footnotetext{
${ }^{1}$ La trilogie regroupe les ouvrages que Michel Henry consacre à une approche du christianisme : C'est moi la vérité. Pour une philosophie du christianisme, Paris, Seuil, 1996 ; Incarnation. Une philosophie de la chair, Paris, Seuil, 2000 ; Paroles du Christ, Paris, Seuil, 2002.

${ }^{2}$ Une seule référence notoire est ici à signaler dans l'article «Archi-christologie »: «Claude Tresmontant a rétabli la datation vraisemblable des Evangiles contre l'exégèse sceptique du XIXe siècle. Il n'est pas possible de présenter ici son argumentation; disons seulement que l'Archichristologie de l'authenticité lui doit beaucoup » (dans Phénoménologie de la vie. IV. Sur l'éthique et la religion, Paris, PUF, « Epiméthée », 2004, p. 115, en note). Par ailleurs, on dénombre d'autres références à Claude Tresmontant dans les notes préparatoires. Cf. Ms A 38-13-27112: « [Michel Henry évoquant les paraboles :] Textes quasi contemporains du Christ. Les synoptiques ne se sont pas recopiés l'un l'autre mais proviennent d'une même source ou de séries de sources tout à fait [illisible] une autre source est Jean. Donc l'Evangile de Jean glose sur un texte originaire probablement écrit dicté par Jean (cf. Cl. Tresmontant)»; Ms A 39-3-27587 : « Note historique. Remarques historiques concernant les Paroles du Christ et découlant d'elles-Luc 14, 35 (et le contexte) et dossier im[manence] Vie in vie. "Celui d'entre vous qui ne renonce pas à tout ce qui lui appartient ne peut pas être mon disciple» in (recueil Paroles antérieures aux Synoptiques). Paul « qu'aies-tu que tu n'aies reçu- Tradition johannique (cf. Tresmontant)». Par ailleurs, on tient à souligner que la référence à Claude Tresmontant se lit implicitement dans corpus henryen en de très nombreux endroits, spécialement quand Michel Henry traite de la question du statut des Ecritures et notamment à l'occasion de la théorie du verbe esquissée dans Paroles du Christ : " On peut donc admettre, contrairement aux contrevérités de l'exégèse positiviste, pseudo-historique et athée du XIXe siècle, que les paroles du Christ n'ont rien à voir avec les inventions de communautés chré-
} 
nologie de la vie. Malgré la distance qui sépare cette dernière démarche de la «philosophie chrétienne » dont se revendique l'auteur du Christ hébreu, notre volonté est de saisir les thèmes qui sont communs à ces deux approches du christianisme. À cet égard, il y a lieu de comprendre comment Claude Tresmontant et Michel Henry ont une préoccupation commune dont l'exigence peut se formuler comme suit : préserver la transcendance de la parole de Dieu tout en soulignant sa nécessaire immanence à l'humanité passe inéluctablement par la singularisation de la pensée judéochrétienne dans sa différence radicale d'avec les schèmes de la philosophie " grecque ». Que le christianisme possède dans et par lui-même les ressources d'une « ontologie » propre, voilà finalement la thèse qui justifie cette étude que nous déploierons en trois temps. Ceux-ci correspondent aux concepts fondamentaux du monde biblique, en l'occurrence, ceux de Création, de Révélation et enfin d'Ecritures ${ }^{1}$.

\section{1/ Création continue et engendrement}

Dès 1953, dans son premier ouvrage publié2, Claude Tresmontant souligne l'incompatibilité de la «pensée hébraïque » avec la philosophie grecque ${ }^{3}$. C'est au niveau de la conception de la genèse du réel que se lit l'écart entre ces deux ontologies : « Penser la genèse du réel comme une création, comme une opération positive, souligne Tresmontant, est une originalité de la tradition biblique $»^{4}$. Sur ce point, «la pensée hébraïque va exactement en sens inverse de la pensée grecque $»^{5}$. Si la

tiennes tardives. Elles s'offrent à nous à titre de documents authentiques » (Paroles du Christ, op. cit., p. 11). Il conviendra de montrer comment cette référence renvoie nécessairement au rapport que Claude Tresmontant entretient aux Ecritures et notamment au climat de méfiance qu'il fait régner à l'égard des exégètes - Bultmann notamment - qui remettent en question le caractère divin des paroles du Christ. De la sorte on peut citer par exemple : «L'école de Bultmann est partie du présupposé qu'en somme n'importe qui pouvait, au cours du premier siècle de notre ère, $[\ldots]$ pouvait se permettre de malaxer, de transformer, de retoucher, de tripoter les paraboles du Seigneur et éventuellement d'inventer des histoires, d'inventer des paraboles, et que les communautés chrétiennes du premier siècle acceptaient ce genre de tripotage. Présupposé hautement invraisemblable quand on connaît le respect infini des frères et des sœurs du judaïsme du premier siècle de notre ère, $[\ldots]$ pour les Livres saints, que personne n'a le droit de toucher, encore moins de retoucher » (Claude Tresmontant, Le Christ hébreu. La langue et l'âge des Evangiles, Paris, O.E.I.L., 1983, p. 206-207.)

${ }^{1}$ À mesure de nos recherches, il nous a paru important d'organiser notre étude en suivant l'ordre du triptyque de Claude Tresmontant qui traitent des trois moments évoqués dans les ouvrages suivants : Comment se pose aujourd'hui le problème de l'existence de Dieu, Paris, Seuil, 1966 ; Le problème de la révélation, Paris, Seuil, 1969 ; L'enseignement de Ieschoua de Nazareth, Paris, 1970. On peut aisément mettre en parallèle ces moments chez Michel Henry qui correspondent aux concepts d'engendrement, de révélation et de « paroles du Christ».

${ }^{2}$ Claude Tresmontant, Essai sur la pensée hébraïque, Paris, Cerf, « Lectio divina », 1953.

${ }^{3}$ Ibid., p. 11.

${ }^{4}$ Ibid., p. 14 
seconde se caractérise par un geste négatif dans lequel le réel sensible est obtenu à l'occasion d'une "dégradation ", en revanche, la première est le fruit d'un acte positif et relève d'une « ascension ». Autrement dit, dans le premier cas, la genèse du réel est la conséquence d'une aliénation, dans le second, par contre, cette action est caractérisée par l'idée de croissance. À cet égard, on doit constater que Claude Tresmontant utilise la philosophie de Bergson pour donner un sens à la spécificité de la « pensée hébraïque »:

Ses conclusions [celles de Bergson] vont en sens inverse de la métaphysique antique [...]. [Or] $\mathrm{Ce}$ renversement du mouvement profond de la pensée hellénique [...] coïncide avec l'orientation naturelle et le sens de la pensée hébraïque, qui, elle aussi, va à contre-courant par rapport aux habitudes de penser de l'intellect grec. ${ }^{1}$

Or Michel Henry a également la volonté de singulariser le christianisme par rapport à la pensée grecque ${ }^{2}$. Selon lui, l'homme chrétien est défini par le « cœur», terme « qui revient si souvent dans les Evangiles [désignant] [...] ainsi la réalité de l'homme comme essentiellement affective, ce qu'elle est en vérité $»^{3}$. Dans cette perspective, cette conception de l'humanité s'oppose en tout point à la pensée hellénique puisque «sur le plan historique, souligne Michel Henry, elle a ébranlé l'horizon de pensée qui était celui des Grecs pour lesquels l'homme est un être rationnel $»^{4}$. Remarquons également que cette différence abyssale recoupe l'abîme qui sépare le «langage humain » de la Parole de Dieu, dès lors que cette dernière est caractérisée par l'affectivité. D'une part, il s'agit en effet d'affirmer, par une lecture de Jn 1, 4 — « Ce qui fut en lui [dans le Verbe] était la vie » - qu' «avec le christianisme surgit l'intuition inoü̈e d'un autre Logos », une parole, souligne Henry, «dont la possibilité est la Vie elle-même et dans laquelle la vie parle d'ellemême $»^{5}$. D'autre part, de reconnaître en cette Parole une «Affectivité primitive en laquelle la vie s'éprouve soi-même immédiatement $»^{6}$.

\footnotetext{
${ }^{1}$ Ibid., p. 15. taine légitimité.

${ }^{3}$ Michel Henry, Paroles du Christ, op. cit., p. 19.

${ }^{4}$ Ibid.

${ }^{5}$ Ibid., p. 94

${ }^{6}$ Ibid., p. 98
}

${ }^{2}$ Pour se prémunir de toute objection, il convient ici d'évoquer la manière dont Claude Tresmonant envisage les rapports entre le christianisme et le judaïsme. Le premier constitue, d'après lui, une radicalisation du second: "Le christianisme continue le judaïsme et l'achève, non pas en l'adoucissant, en l'émasculant, en le lénifiant, mais au contraire en augmentant, en poussant à l'extrême, l'exigence, les exigences contenues en germe dans la Torah et les prophètes » (L'enseignement de Ieschoua de Nazareth, op. cit., p. 139). Nous reviendrons sur le sens de cette thèse. Néanmoins, il faut constater que si le christianisme est une radicalisation du judaïsme, alors penser la singularité de la pensée hébraïque vaut également pour qualifier la logique de la Nouvelle Alliance. Par conséquent, le rapprochement avec la démarche de Michel Henry acquiert une cer- 
À cet égard, il est intéressant de constater que selon Claude Tresmontant, la différence entre «pensée hébraïque » et métaphysique hellénique recoupe également la distinction entre l'homme et Dieu : «Analyser les positions de la pensée hébraïque biblique, c'est faire l'inventaire de ce qui est contenu, impliqué dans l'idée de création, c'est la distinguer de ce qui n'est pas elle (la fabrication, qui est un faire proprement humain) $\|^{1}$. Or, conformément à sa démarche, c'est encore à Bergson que Tresmontant emprunte cette distinction ${ }^{2}$, articulée à une réflexion destinée à circonscrire la singularité du concept de création au prisme de la triade "nouveauté », « création continuée », « durée », telle qu'on la retrouve dans L'évolution créatrice. Celle-ci est mobilisée pour dégager le sens présent dans le texte biblique qui rend possible l'idée d'une "pensée hébraïque ».

Même si ce n'est pas le lieu de nous interroger sur la légitimité de l'usage spécifique de ces concepts dès lors qu'ils sont mêlés aux Ecritures, il convient néanmoins d'en rendre compte. De la sorte, évoquant de nombreux passages des Ecritures, Tresmontant insiste sur le fait que, tant dans l'Ancien ${ }^{3}$ que dans le Nouveau testament, «créer, c'est créer du nouveau ». Survient alors un geste herméneutique puissant : «Cette intuition du nouveau, cette sensibilité à la signification métaphysique du radicalement nouveau, qui est le caractère même de l'acte de création, a été la vue vive de Bergson $»^{5}$. Partant, c'est la critique conjointe du mécanisme et du

${ }^{1}$ C. Tresmontant, Essai sur la pensée hébraïque, op. cit., p. 25. Cette distinction est fondamentale. On la retrouve constamment dans les propos de Tresmontant. Par exemple : «Habitués à voir de nos yeux l'ouvrier, l'artisan qui rassemble les matériaux pour construire la maison ou la machine, nous avons du mal à comprendre et à discerner le créateur qui procède de l'intérieur et qui demeure caché à nos yeux » (Comment se pose aujourd'hui le problème de l'existence de Dieu, op. cit., p. 264). Autre exemple : «L'idée de création est extrêmement difficile à penser, peut-être la plus difficile des idées métaphysiques, tout simplement car nous n'avons pas l'expérience personnelle de la création de l'être. Nous avons l'expérience constante universelle de la fabrication, par laquelle nous arrangeons des matériaux, du bois, du fer ou de la pierre, pour façonner un objet quelconque » (Le problème de la révélation, op. cit., p. 57).

${ }^{2}$ Evoquant la question de l'élan vital, Bergson fait part d'une distinction, non pas entre « fabrication » et « création », mais bien entre « organisation » et « fabrication»: « Mais c'est ce que nous avons beaucoup de peine à comprendre, parce que nous ne pouvons nous empêcher de nous représenter l'organisation comme une fabrication [...]. La fabrication va [...] de la périphérie au centre ou, comme diraient les philosophes, du multiple à l'un. Au contraire, le travail d'organisation va du centre à la périphérie. Il commence en un point qui est presque un point mathématique, et se propage autour de ce point par ondes concentriques qui vont toujours s'élargissant » (L'évolution créatrice, Paris, PUF, « Bibliothèque de philosophie contemporaine », 1940, p. 93.) Dans la suite du texte, on se permet de faire systématiquement référence à cette version du texte qui correspond à celle qu'utilise Tresmontant.

${ }^{3}$ Voici quelques références bibliques qui font référence à la «nouveauté » caractérisant le concept de création. On se permet de les restituer dans la traduction de l'Ecole biblique de Jérusalem. Ainsi, par exemple, dans l'Ancien Testament: «Yahvé créée du nouveau sur la terre » $(\mathrm{Jr} 31,32)$. Pour ce qui est de la Nouvelle Alliance : « la circoncision n'est rien, ni l'incirconcision ; il s'agit d'être une créature nouvelle » $(\mathrm{Ga} 6,15)$.

${ }_{5}^{4}$ C. Tresmontant, Essai sur la pensée hébraïque, op. cit., p. 27.

${ }^{5}$ Ibid. 
finalisme radical ${ }^{1}$ qui est utilisée par Tremontant pour qualifier la " nouveauté » qui caractérise l'acte créateur du Dieu d'Abraham. Ce régime d'innovation renvoie aussitôt Tresmontant vers le concept bergsonien de «création continue » par contraste avec le concept cartésien de «création continuée». Descartes ne rend pas l'idée de mouvement inhérente à l'acte de création en ce sens qu'une fois créée, «la chose n'a besoin que d'être conservée $»^{2}$. En revanche, Bergson, serait seul capable de restituer l'idée biblique de genèse : «dans l'univers biblique, et chez Bergson, souligne Tresmontant, "la création n'apparaît plus simplement comme continuée, mais comme continue" ». Ce sont donc les propos concernant le couple «mécanisme et conceptualisme » qui donnent un sens à la formule johannique relatant les dires du Christ selon lesquels « Mon Père est à l'œuvre jusqu'à présent et j'œuvre moi aussi » (Jn 5, 17). Ceux-ci évoquent en retour que « L'acte de création continue d'opérer, d'inventer génialement $»^{3}$.

Claude Tresmontant, toujours en reprenant les thèses de Bergson, mais cette fois celles de La pensée et le mouvant, souligne que cette idée est ce qui définit la temporalité biblique dans sa différence avec la pensée hellénique. En effet, s'il est une chose que n'accepte pas la philosophie, c'est cette création continue d'imprévisible nouveauté. En d'autre termes, ce que les Grecs ignorent c'est l'idée même de durée. D'une part, parce que « plus ou moins platoniciens, souligne Bergson, ils [les Grecs] se figuraient que l'Etre était donné une fois pour toutes, complet et parfait, dans l'immuable système des Idées $»^{4}$. Ce qui, d'autre part, mènerait à une conception strictement négative du temps, c'est-à-dire du « monde » :

Le monde qui se déroule à nos yeux ne pouvait donc rien y ajouter [à l'Etre] ; il n'était au contraire que diminution, dégradation; ses états successifs mesureraient l'écart croissant ou décroissant entre ce qu'il est, ombre projetée dans le temps, et ce qu'il devrait être, Idée assise dans l'éternité [...]. C'est le Temps qui aurait tout gâté. ${ }^{5}$

Or, précisément, cette idée contredit la logique fondamentale de « l'univers biblique »; car selon la belle formule de Claude Tresmontant, «l'hébreu a l'amour du sensible $»^{6}$. Pour preuve de celui-ci, on peut invoquer l'authentique dignité du réel qui est attestée par le Créateur à même son acte de création : «...et Dieu vit que cela était bon » $(\mathrm{Gn} 1,17)$. D'où l'idée centrale de l'Essai sur la pensée hébraïque, de reprendre le concept de durée pour restituer cette logique qui se situe

\footnotetext{
${ }^{1}$ Bergson souligne bien que : «Dès que nous sortons des cadres où le mécanisme et le finalisme radical enferment notre pensée, la réalité nous apparaît comme un jaillissement ininterrompu de nouveauté $[\ldots]$... (L'évolution créatrice, op. cit., p. 47.)

${ }^{2}$ C. Tresmontant, Essai sur la pensée hébraïque, op. cit., p. 28.

${ }^{3}$ Ibid.

${ }_{5}^{4}$ H. Bergson, La pensée et le mouvant, Paris, PUF, 1969, p. 115.

${ }^{5}$ Ibid.

${ }^{6}$ C. Tresmontant, Essai sur la pensée hébraïque, op. cit., p. 56.
} 
à l'opposé de la pensée hellénique. En effet, plus que tout autre, ce concept est capable de restituer une positivité au Temps qui inverse l'idée d'une catagénèse. D’une part, souligne Bergson, « La durée [...] est création continuelle, jaillissement ininterrompu de nouveauté »'. D'autre part, poursuit-il, «L'univers dure [...] durée signifie invention, création de formes, élaboration continue de l'absolument nouveau $»^{2}$. C'est de la sorte que Claude Tresmontant peut finalement dire que «Dieu a le temps $»^{3}$

Dans l'élaboration de la phénoménologie matérielle, Michel Henry tient ses distances avec la philosophie de Bergson. Et s'il est tout à fait possible de rapprocher ces auteurs ${ }^{4}$, ceci n'a rien d'une évidence. L'une des thèses de Michel Henry consiste de fait à soutenir que "la vie est incréée » et qu'en tant qu' «étranger à la création, étranger au monde, tout procès conférant la Vie est un procès de génération de la Vie absolue comme génération en elle de son Verbe $»^{5}$. Pourtant, le geste qui consiste à suspendre toute création, pour saisir l'essence de la manifestation comme un processus de génération capable de constituer par lui-même une expérience, ne semble pas contredire l'idée que la vie ainsi décrite puisse être compatible avec le concept de durée. Même si un traitement plus radical de cette question est requis, il ne fait aucun doute que la conception henryenne de l'essence comme immanence possède les traits d'un processus poétique dont les modalités pourraient être comprises comme étant celles d'une création continue. Ceci est patent dès lors que Michel Henry souligne, à l'occasion d'un commentaire de Ephésiens 4, 6, que cette vie qui définit Dieu est « essence agissante, se déployant avec une force invincible, source de puissance, puissance d'engendrement immanente à tout ce qui vit $»^{6}$. Cependant, en portant cette idée à l'absolu, Michel Henry se démarquerait de la position de Claude Tresmontant, ce dernier tenant en effet pour essentielle la différence entre le monde et Dieu en même temps qu'il circonscrit la portée du concept d'engendrement au Christ.

\footnotetext{
${ }^{1} \mathrm{H}$. Bergson, La pensée et le mouvant, op. cit., p. 9.

${ }^{2} \mathrm{H}$. Bergson, L'évolution créatrice, op. cit., p. 11 . Cette idée d'une genèse créatrice dont la temporalité est celle de la durée est ce qui permet également à Claude Tresmontant de faire une lecture originale de Luc 31,18 et d'obtenir de la sorte une compréhension de ce en quoi consiste le «Royaume de Dieu »: « Le royaume des cieux est semblable à un grain de sénevé qu'un homme a pris et jeté dans son champ. C'est la plus petite de toutes les graines, mais quand elle a poussé, il est plus grand que les légumes et devient un arbre, de telle sorte que les oiseaux du ciel viennent habiter dans ses branches » (Essai sur la pensée hébraïque, op. cit., p. 76.)

${ }^{3}$ Claude Tresmontant, Problèmes du christianisme, Paris, Seuil, 1980, p. 78.

${ }^{4}$ Cette piste de travail a déjà été envisagée par Camille Riquier qui tente un lien entre ces deux auteurs via une source commune, en l'occurrence, Maine de Biran. On se permet de renvoyer le lecteur à son étude tout à fait déterminante : «Henry, Bergson et la phénoménologie matérielle » dans Studia Phaenomenologica, 9 (2009), p. 157-172.

${ }^{5}$ M. Henry, Paroles du Christ, op. cit., p. 107.

${ }^{6} \mathrm{M}$. Henry, C'est moi la vérité, op. cit., p. 72. Saint Paul appelant à l'unité dit bien dans sa lettre : « un seul Seigneur, une seule foi, un seul baptême ; un seul Dieu et Père de tous, qui est au-dessus de tous, par tous et en tous » (Ephésiens 4, 5-6).
} 
Distinguer Dieu du monde, c'est avoir la volonté, dit Tresmontant, de préserver l'orthodoxie de la doctrine chrétienne de la création. Concrètement, il s'agit de refuser toute espèce d' ' égoïsme transcendantal » pour faire droit à la logique du don. Le premier point de vue consiste à soutenir la thèse selon laquelle Dieu crée l'univers pour répondre à un besoin motivé par un manque. Or, si tel est le cas, l'absolu est une idée contradictoire. S'il lui manque quelque chose, c'est précisément parce qu'il ne constitue aucune plénitude de par lui-même, ce qui revient à lui ôter le statut auquel il prétend. Réfuter ce propos présuppose qu'on limite la portée du concept de durée au seul monde créé. Si tel est le cas, alors d'une part, l'on doit reconnaître, comme le fait Tresmontant que « la durée réelle mesure une création en train de se faire ${ }^{1}$. D'autre part, et parce que l'absolu ne peut connaitre aucun accroissement évoquant l'idée d'un manque qui lui serait inhérent, on est contraint de tenir que « Dieu est plénitude absolue avant la création, indépendamment de la création $»^{2}$. Dans ce cadre, le fait que Dieu crée le monde ne peut strictement rien lui apporter puisqu'il est une plénitude à quoi rien ne fait défaut. Partant, la création du monde est de l'ordre du don parce qu'elle ne vient combler aucun désir imputé à Dieu : "C'est parce que Dieu est plénitude en lui-même, souligne Tresmontant, parce qu'il n'a pas besoin à proprement parler de la création pour se réaliser, c'est pour cela que la création est un don $»^{3}$.

Michel Henry semble être en porte-à-faux avec cette logique en ce sens que son approche phénoménologique du christianisme suggère l'idée d'un l'ipséisme transcendantal susceptible de dépasser les deux logiques dont il vient d'être question en les réarticulant sur le plan originaire de la passivité. Ce qu'il convient cependant de bien retenir, c'est que le phénoménologue, en absolutisant l'idée d'une essence agissante, évacue la possibilité d'un Dieu qui précéderait toute création. L'incréé henryen est déjà poétique. ${ }^{4}$ Par conséquent, l'immanence possède une temporalité propre de laquelle ne s'exclut à aucun moment le Verbe. À cet égard, le rejet de la création tel qu'il est pensé dans le cadre de la phénoménologie de la vie pourrait paradoxalement signifier sa réhabilitation sous les espèces d'une plénitude en tant que création continue.

Parce qu'il souhaite toujours sauvegarder la logique du don, Tresmontant rappelle par ailleurs qu'on trouve, dans la métaphysique chrétienne, une insistance sur le fait que « le monde est créé, et non engendré, c'est-à-dire que le monde n'est pas fait de

\footnotetext{
${ }^{1}$ Ibid., p. 13

${ }^{2}$ C. Tresmontant, Le problème de la révélation, op. cit., p. 15.

${ }^{3}$ Ibid.

${ }^{4}$ Pour une explication exhaustive du contresens selon lequel la vie, chez Michel Henry, serait " intemporelle », nous renvoyons le lecteur à G. Jean, "La subjectivité, la vie, la mort » dans Revue Internationale Michel Henry, n³ (2012), p. 46-61.
} 
la substance divine $»$. Or, ce fait possède une répercussion anthropologique majeure puisqu'il n'est plus possible de défendre la thèse gnostique selon laquelle l'homme serait fait de la substance divine. La théologie biblique rejette d'ailleurs, poursuit Tresmontant, l'idée même que «l'homme aurait été créé, ou plutôt engendré avec un élément de la substance divine »². En revanche, «le Fils, le Logos, lui n'est pas créé, il est engendré $»^{3}$.

En décrivant ce qu'il nomme la condition de Fils, Michel Henry semble se rapprocher d'une certaine forme de gnosticisme. Le phénoménologue esquisse néanmoins une théorie d'après laquelle le Verbe ne se confond pas avec l'homme. Dieu est pensé sous un concept fort d'auto-affection qui désigne cette capacité de produire soi-même le contenu de sa propre affection : «Pour passive que soit cette épreuve que la vie fait constamment d'elle-même dans son étreinte pathétique, souligne Michel Henry, elle n'en est pas moins produite par la vie elle-même, et c'est cette génération par soi de la vie qu'indique le concept fort de l'auto-affection $»^{4}$. Par contre, l'homme est en régime d'auto-affection «faible » désignant le fait que tout en produisant le contenu de sa vie, il n'est cependant pas l'auteur de la condition par laquelle il lui advient: «je m'éprouve moi-même sans être la source de cette épreuve $\aleph^{5}$. Cependant, cette distinction n'altère en rien le fait que l'homme et Dieu partagent la même essence puisque le premier se trouve déterminé par le second: «Le Soi ne s'auto-affecte que pour autant que s'auto-affecte en lui la Vie absolue $»^{6}$.

\section{2/ Révélation et résistance à l'information}

Malgré la distance qui sépare la démarche de Claude Tresmontant de la phénoménologie de la vie proposée par Michel Henry, ces deux approches du christianisme convergent néanmoins de façon tout à fait étonnante dans leur manière d'affirmer la transcendance de la Parole de Dieu dès lors que sa présence se déploie en l'homme sous les espèces de la durée. C'est que création et révélation y sont intimement liées. À cet égard, l'extrême méfiance de Claude Tresmontant par rapport à l'exégèse ${ }^{7}$ ne

${ }^{1} \mathrm{C}$. Tresmontant, La métaphysique du christianisme et la naissance de la philosophie chrétienne. Problèmes de la création et de l'anthropologue des origines à Saint Augustin, Paris, Seuil, 1961, p. 196.

${ }_{2}^{2}$ C. Tresmontant, La métaphysique du christianisme, op. cit., p. 196.

${ }^{3}$ Ibid., p. 197.

${ }_{5}^{4} \mathrm{M}$. Henry, C'est moi la vérité, op. cit., p. 135.

${ }^{5}$ Ibid., p. 136.

${ }^{6}$ Ibid.

${ }^{7}$ Le rapport de Tresmontant à l'exégèse biblique est ambigu. Malgré le fait qu'il loue la critique biblique, il dresse néanmoins un portrait peu flatteur de l'exégète. Ainsi en est-il pour Renan par exemple. Dans Le problème de la révélation (1969) le principal reproche qui lui est fait est d'avoir injustement remis en cause l'idée de "l'inspiration surnaturelle » en posant un faux dilemme selon lequel les Ecritures sont soit humaines soit divines. Ayant démontré l'évidence du fait qu'elles sont 
doit pas nous faire oublier qu'il trouve dans la «critique biblique » les ressources d'une analogie entre création et révélation. Cette critique aurait provoqué une « révolution » dans notre manière de lire la Bible qui consiste « dans le fait que la Révélation [...] ne se présente plus à nous comme un bloc donné, pour une grande part, une fois pour toutes $[. .$.$] mais comme développement progressif \rangle^{1}$. La distinction de couches rédactionnelles dans l'écriture des livres du Pentateuque pose en effet le problème de sa composition. Ce qui pousse à conclure que « la révélation n'a pas été donnée d'un seul coup, sur le Mont Sinaï. La révélation a été communiquée progressivement $»^{2}$. Notons que Michel Henry semble être tributaire de cette manière de voir les choses puisqu'il dit: «C'est le Christ lui-même qui va révéler qui il est. Révélation [qui est] progressive [...] si l'on s'en tient au texte ». ${ }^{3}$

Pour Claude Tresmontant, le fait que la Révélation soit progressive renvoie immanquablement à son fond bergsonien. L'argument est le suivant : la science montre que la création ne se fait pas d'un seul coup mais progressivement. Ainsi en est-il par exemple pour ce qui est de l'évolution du genre humain. Ceci implique donc qu'il « faut se représenter la création comme [...] un processus progressif, comme une invention qui a duré » ${ }^{4}$. Et Bergson de ressurgir dans la foulée de cette digression : «l'évolution se présente à nos yeux comme la création en train de se faire ; c'est ce qu'avait vu Bergson et ce qu'il avait exprimé dans son ouvrage : l'Evolution créatrice $»^{5}$. C'est bien cette thèse qui fonde l'idée d'un progrès dans la Révélation puisque Tresmontant dit finalement : «Eh bien, il en va de même, mutatis mutandis, en ce qui concerne la Révélation $»^{6}$.

Parce qu'il s'agit toujours de préserver la logique du don, il est ici question de distinguer Dieu comme source de l'information et la création qui la reçoit. C'est à ce niveau qu'intervient la figure du prophète. Ce dernier ne se confond pas avec la source de l'information puisque c'est Dieu lui-même qui, par sa Parole crée toute choses : «La création a été effectuée, elle est effectuée et réalisée par la parole de

rédigées par des hommes, Renan refuse donc toute transcendance à la Parole de Dieu. C'est ce que ne peut accepter Tresmontant qui tient, en vertu de sa méthode qui s'appuie sur la théorie des deux natures du Christ, que les Ecritures sont le fruit d'une co-opération entre Dieu et l'homme (cf. p. 41 sq.) Dans Le Christ hébreu (1983), ce même reproche devient complètement caricatural dès lors qu'il est appliqué à Bultmann et la thèse selon laquelle les évangiles auraient été rédigés par des communautés tardives ce qui remettrait en cause la messianité des paroles attribués à Jésus : «L'école de Bultmann présuppose ce qui est en question : à savoir que les quatre Evangiles sont des rédactions tardives postérieures à une longue tradition orale, à de longues traditions, à des prédications. Pendant ce temps les communautés chrétiennes ont eu le temps de sécréter les histoires évangéliques et les paraboles évangéliques » (p. 204.) Ce que n'accepte pas Tresmontant, c'est l'idée que les Ecritures soient le fruit d'une rédaction exclusivement humaine.

${ }^{1}$ C. Tresmontant, Le problème de la révélation, op. cit., p. 33.

${ }^{2}$ Ibid., p. 34 ; nous soulignons.

${ }^{3}$ M. Henry, Paroles du Christ, op. cit., p. 59-60.

${ }^{4} \mathrm{C}$. Tresmontant, Le problème de la révélation, op. cit., p. 35.

${ }^{5}$ Ibid., p. 36.

${ }^{6}$ Ibid. 
Dieu. C'est la doctrine constante dans la tradition hébraïque $»^{1}$. Dans cette perspective, Tresmontant souligne que «le monde sensible est langage, il a été créé par la parole $»^{2}$. Partant, c'est au réel qu'est restituée toute la dignité qui légitime l'amour du sensible dont témoigne le monde biblique. À cet égard, le premier verset du Prologue johannique est relu dans le sillage de la Genèse. Le fait que « Dieu vit que cela était bon » renvoie coup sur coup à l'acte créateur $($ Genèse 1,1$)$ et aux propos de saint Jean (Jean 1, 1) qui insiste alors sur le fait que c'est dans et par le Verbe qu'advient la création (Jean 1,3). L'intervention du Logos dans l'acte créateur est rendue chez Tresmontant par une expression qui, une nouvelle fois, évoque ses présupposés herméneutiques : «au commencement était l'information créatrice, elle était auprès de Dieu, elle venait de lui, et a été créé par elle et rien de ce qui a été créé n'a été créé sans elle $»^{3}$.

La Révélation, c'est donc le fait que la création s'effectue à l'occasion d'une Parole créatrice qui appartient à Dieu. Mais le destin de celle-ci se trouve soumis à une double perspective dès lors qu'elle est reçue et interprétée par l'humanité : soit l'information se trouve «dégradée », soit celle-ci se trouve « continuée». La première attitude évoque ce que Tresmontant nomme la «scolastique » : «La scolastique, souligne-t-il, c'est la répétition de la pensée du maitre, mais une répétition n'est pas une création. Une pensée répétée n'est pas forcément une pensée repensée. C'est le plus souvent une pensée dégradée ${ }^{4}$. On comprend alors comment se rejoue ici une conception typiquement grecque de la temporalité faisant que l'origine ne peut alors manquer de se perdre à même sa réception: "Dans sa transmission, le message primitif a perdu de sa richesse. Son information a diminué $»^{5}$. Concernant l'histoire de la Révélation, l'exact envers de ce travers est incarné par la figure du prophète. Ceci permet d'atténuer la froideur de la logique du don selon laquelle, l'homme ne contribuerait en rien l'édification de l'absolu. À cet égard, Tresmontant souligne que «dans l'histoire du prophétisme hébreu, ce qui est remarquable, c'est

${ }^{1}$ C. Tresmontant, Le Christ hébreu, op. cit., p. 219.

${ }^{2}$ C. Tresmontant, Essai sur la pensée hébraïque, op. cit., p. 56.

${ }^{3}$ C. Tresmontant, Problèmes du christianisme, op. cit., p. 52 ; nous soulignons. L'influence de Bergson dans cette interprétation tout à fait originale du premier verset du quatrième évangile est encore plus palpable si on considère sa version dans le cadre de la traduction intégrale de l'Evangile de Jean que propose Claude Tresmontant. Nous reviendrons sur les modalités de cette entreprise dans la troisième partie de notre étude. Voici le passage qui doit intéresser le lecteur: « au commencement était le parler et le parler était à dieu et dieu il était le parler» (Evangile de Jean, trad. Tresmontant, Paris, F.-X. de Guibert, 1994, p. 17). Or, voici comment l'auteur justifie l'usage de du terme " parler » en lieu et place de «Parole»: «Toute création dans l'Univers et dans la nature est l'œuvre d'une parole créatrice. Rien n'est créé sans cette parole créatrice. C'est l'acte de dire qui est au principe de toute création. Le mot parole en français présente l'inconvénient de désigner ce qui est dit, la chose qui est dite. Dans Genèse 1, c'est l'acte même de dire qui est à l'origine de chaque création nouvelle. La parole de Dieu est créatrice. Ce qui est dit, c'est l'être créé. L'acte de dire, c'est l'acte de créer » (p. 121).

${ }^{4}$ C. Tresmontant, Problèmes du christianisme, op. cit., p. 248.

${ }^{5}$ C. Tresmontant, Le problème de la révélation, op. cit., p. 82. 
que l'information augmente au cours du temps et devient de plus en plus riche ${ }^{1}$. Le rabbi Ieschouah est à compter parmi ceux-ci puisqu'en en héritant de la Torah, il radicalise ses exigences qui passent d'un plan extérieur à un plan intérieur, c'est ce qui expliquerait le sens de Matthieu 5, 17: «Ne pensez pas que je sois venu détruire la Torah ou les prophètes. Je ne suis pas venu détruire, mais accomplir, achever ». Mais il faut rester prudents. En effet, à côté de ceux qui à l'image du Christ sont capables de continuer et d'augmenter le message, Tresmontant met en garde contre les « faux-prophètes $»^{2}$. Ce sont les imposteurs : «Le faux prophète est celui qui parle et enseigne "à partir de son propre cœur". Dieu ne l'a pas envoyé, Dieu ne lui a pas parlé objectivement. Il obéit, dirait-on dans le galimatias moderne, à sa propre "subjectivité" " ${ }^{3}$. Au-delà de la violence de cette thèse, un élément doit nous intéresser - le critère qui distingue le « vrai » prophète du « faux », car c'est à partir de ce point que l'on retrouve l'approche henryenne du christianisme.

Ce qui permet de distinguer le vrai prophète c'est le fait qu'il porte en lui la Parole créatrice. Cependant, la présence de celle-ci ne se mesure réellement qu'à la violence exercée à l'égard de celui qui la porte. À cet égard, Tresmontant parle d'une résistance à l'information signifiant un refus par le monde de la nouveauté inhérente au témoignage prophétique. Il convient de distinguer deux types d'entraves à la Parole. Une résistance «passive » en vertu de laquelle ceux qui l'écoutent n'y sont pas attentifs. Mais c'est surtout l'idée que ce message puisse faire l'objet d'un refus explicite qui doit nous intéresser, car dans ce cas, « il s'agit d'une résistance active, qui peut aller jusqu'au meurtre de l'enseigneur parce qu'il s'agit d'une détestation »". C'est d'ailleurs dans cette perspective qu'est conféré un sens à Luc 13, 34 : «Jérusalem, Jérusalem, toi qui tues les prophètes et qui lapides ceux qui te sont envoyés ». L'objet de cette résistance n'est rien moins que la Parole dans la nouveauté qu'elle propose: "C'est toujours la nouveauté du message prophétique, souligne Claude Tresmontant, qui suscite une résistance d'autant plus acharnée et violente que le message est plus nouveau $»^{5}$. Celle-ci est donc bien le signe d'une parole en régime de création continue. Mais c'est surtout le témoin qui permet de discerner l'authenticité, c'est-à-dire la transcendance de la Parole portée par le prophète dans ce qu'elle a d'irréductible au langage humain. C'est dans cette perspective que Tresmontant souligne que «Cette résistance violente au prophétisme est un signe d'authenticité. Des filous, des imposteurs, ne s'amuseraient pas à encourir la persécution et la mort violente pour le plaisir d'enseigner, à contre-courant, la parole

${ }^{1}$ C. Tresmontant, Problèmes du christianisme, op. cit., p. 248-249. Pour un traitement complet de ce thème, nous renvoyons le lecteur à $\mathrm{C}$. Tresmontant, Le prophétisme hébreu, Paris, Gabalda, 1982.

${ }^{2}$ Pour cette problématique, cf. C. Tresmontant, L'enseignement de Ieschoua de Nazareth, op. cit., p. 139.

${ }^{3}$ C. Tresmontant, Le problème de la révélation, op. cit., p. 144.

${ }^{4}$ C. Tresmontant, Problèmes du christianisme, op. cit., p. 250.

${ }^{5}$ Ibid. 
du Dieu invisible $»^{1}$. Or, poursuit-il, s'il est « une preuve que cet enseignement ne vient pas des hommes, c'est la résistance qu'il rencontre parmi les hommes auxquels il s'adresse $»^{2}$.

Le Christ est un exemple limite de cette violence déployée à l'encontre de celui porte la Parole de Dieu puisqu'il est ce Verbe en tant que tel. Tresmontant le considère d'ailleurs comme "la plénitude de l'information créatrice » ${ }^{3}$. L'un des aspects $\mathrm{du}$ christianisme sur lequel Michel Henry revient très souvent est précisément ce refus qui mène à la condamnation du Christ. Le thème de la haine du Verbe est en effet omniprésent dans Paroles du Christ: "Tout au long de sa vie publique, le Christ s'est heurté à l'incompréhension de ses auditeurs » et spécialement à celle « des plus retors et des hypocrites dont l'hostilité, qui s'est très vite changée en haine, devait le poursuivre jusqu'à la fin $»^{4}$. L'objet de cette détestation n'est rien moins que la «Vraie lumière incarnée dans le Christ ». Laissons pour l'instant de côté l'influence probable de Claude Tresmontant sur la lecture henryenne de Jn 1, 10-11 : «Il était dans le monde, et le monde fut par lui, et le monde ne l'a pas reconnu. Il est venu chez lui, et les siens ne l'ont pas accueilli ». Il faut d'abord envisager un lien explicite entre ces deux auteurs qui nous invite à considérer le thème de la persécution du Christ qui, en un sens, atteste une première fois de la transcendance de sa Parole par rapport au langage humain.

La seule et unique référence explicite de Michel Henry à l'œuvre de Claude Tresmontant est un article dans lequel le premier pose les jalons de ce qu'il appelle une "Archi-christologie ${ }^{6}$. Sa thèse consiste à dire que toute christologie présuppose une «archi-christologie» laquelle est «inscrite dans la parole même des Ecritures $\gg^{7}$. Une question se présente : comment est-il possible d'attester la validité de la christologie intrinsèque au discours que le Christ tient sur lui-même : "Comment la reconnaître et s'en assurer? " ${ }^{8}$. Cette tâche est celle qui caractérise une "archichristologie de l'authenticité »" ${ }^{9}$. L'influence de Tresmontant y est patente en ce sens que Michel Henry reconnaît toute sa dette à l'égard de cet auteur : « Il n'est pas possible de présenter ici son argumentation [celle de Tresmontant], disons seulement que l'Archi-christologie de l'authenticité qui suit lui doit beaucoup $»^{10}$. Le terme même d'authenticité utilisé par le phénoménologue nous semble provenir de la théo-

${ }^{1}$ C. Tresmontant, Le problème de la révélation, op. cit., p. 144 ; nous soulignons.

${ }^{2}$ Ibid., p. 160.

${ }^{3}$ C. Tresmontant, Le Christ hébreu, op. cit., p. 208.

${ }^{4}$ M. Henry, Paroles du Christ, op. cit., p. 115.

${ }^{5}$ Ibid., p. 125.

${ }^{6}$ M. Henry, «Archi-christologie », art. cit., p. 113-129. Cet article est paru une première fois dans dans Communio, 22, n²-3 (1997), p. 195-212.

${ }^{7}$ Ibid., p. 114.

${ }^{8}$ Ibid.

${ }^{9}$ Ibid., p. 115.

${ }^{10}$ Ibid. 
rie de la résistance à l'information. Celle-ci est d'autant plus intéressante que Tresmontant, dans le Christ hébreu, l'applique à la figure de l'Evangéliste Jean. D'une part, en soulignant la proximité entre Jean et le Christ. D'autre part, en soulignant le contexte de "terreur» qui entoure la rédaction du quatrième évangile. Voyons comment Michel Henry se réapproprie ces deux thèses.

Pour Tresmontant, il s'agit premièrement d'affirmer que « le quatrième Evangile est un livre de haute théologie, l'œeuvre d'un théologien qui appartenait au haut sacerdoce hébreu »' ${ }^{1}$. Partant, c'est la datation de cet évangile ainsi que l'âge de celui qui l'a rédigé qui connaissent une inversion par rapport aux thèses de l'exégèse biblique concernant cette même problématique :

Il suffit de lire ou de relire le texte, souligne Tresmontant, pour voir que c'est du reportage pris sur le vif. Cela a été noté le jour même, sur le champ, ou le lendemain. Le quatrième Evangile n'est pas le livre d'un vieillard âgé de quatrevingt-dix-huit ans. C'est un livre écrit par un homme jeune... ${ }^{2}$

C'est bien cette thèse que Michel Henry reprend à son compte dès lors que, d'une part, il affirme : «Jean [est un] prêtre mystique, théologien éminent de l'entourage du grand prêtre $»^{3}$. D'autre part, il fait sienne l'hypothèse selon laquelle ce dernier aurait noté directement ce que lui avait confié le Christ, savoir qu'il est le Fils de Dieu : «comment ce monologue intérieur [la prière sacerdotale] aurait-il pu nous être transmis si le Christ ne l'avait lui-même pas communiqué à Jean, qui l'entendit, l'écrivit et nous le transmit lui-même à son tour tel qu'il l'avait entendu compris et noté $»^{4}$. Ce qui fait donc le caractère authentique de cette archi-christologie, dont le matériel est notamment l'Evangile de Jean, c'est la proximité de son auteur avec le Verbe.

La thèse qui prétend que l'Evangile de Jean serait un document parfaitement contemporain du Christ ${ }^{5}$ fera sourire les détracteurs de Tresmontant ${ }^{6}$, mais il ne faut pas oublier qu'elle se déploie dans le cadre de sa théorie de la résistance à l'information dont le dessein est avant tout d'affirmer le caractère irréductible de la Parole de Dieu au langage humain. Saint Jean a manifestement les traits du prophète puisque ce dernier est contemporain de la parole créatrice. Ceci se vérifie dès lors que Tresmontant insiste sur le contexte dans lequel l'évangéliste a rédigé son texte: « [...] étrange

\footnotetext{
${ }^{1}$ C. Tresmontant, Le Christ hébreu, op. cit., p. 306.

${ }^{2}$ Ibid., p. 289.

${ }^{3}$ M. Henry, « Archi-christologie », art. cit., p. 115.

${ }^{4}$ Ibid. ; nous soulignons.

${ }^{5}$ Tresmontant dit bien : « quelle absurdité, a priori, que de supposer que dans ce milieu lettré, dans ce milieu de scribes, d'hommes du Livre, on ait attendu 70 ans ou 80 ans ou plus pour mettre par écrit ce qui, aux yeux de l'auteur du quatrième Evangile, est le plus important du monde, plus important que ce monde [...]» (Le Christ hébreu, op. cit., p. 289).

${ }^{6}$ Sur ce point, on sera particulièrement attentif à Pierre Grelot, Evangiles et tradition apostolique. Réflexions sur un certain « Christ hébreu », Paris, Cerf, « Apologique », 1984, p. 94-99.
} 
ce document, à savoir le quatrième Evangile, dont on nous répète qu'il est du second siècle ou de la fin du premier, et qui baigne dans une atmosphère de terreur, terreur non pas de la part des autorités romaines [...] mais de la part des autorités de Jérusalem ${ }^{1}$. Notons, une nouvelle fois, que Michel Henry reprend cette thèse à son compte en soulignant que la rédaction de l'Evangile s'inscrit

dans un le contexte tragique qu'elle relate [que nous] saisissons à des signes multiples. L'un des plus significatifs est l'« atmosphère de terreur » dans laquelle cet Evangile fut écrit, à savoir la première et terrible persécution de la communauté chrétienne par les autorités religieuses établies. ${ }^{2}$

Pourquoi Tresmontant insiste-il sur cette persécution si ce n'est précisément pour renforcer la thèse d'une parfaite contemporanéité entre le Verbe et saint Jean? Si celui-ci est pensé à partir de la figure du prophète, cela ne peut que contribuer à la volonté de souligner l'authenticité de son propos puisque « cette résistance violente au prophétisme est un signe d'authenticité $\|^{3}$. De la sorte, si Michel Henry reprend à son compte les thèses de Tresmontant, celle de l'immédiateté de la Parole avec celui qui la porte dont le corolaire est une violence qui rejaillit à son égard, on ne peut alors manquer de souligner que la tâche d'une archi-christologie qui se dit de précisément de l'authenticité ait pour but d'affirmer la transcendance de la Parole de Dieu dans ce qu'elle a d'irréductible au langage humain. Toutefois, Michel Henry, en raison de la duplicité de l'apparaître, souligne l'insuffisance d'une telle démarche puisqu'il tient la thèse selon laquelle : "L'authenticité d'une Archi-christologie [...] n'est pas encore sa vérité $»^{4}$ :

L'existence historique du Christ, de même que les déclarations extraordinaires qu'il n'a cessé de porter sur sa propre personne, pourrait bien être établie selon les critères rigoureux de l'histoire, et ces déclarations n'être pourtant que les divagations d'un exalté ou d'un fou. Preuve en est que beaucoup de ceux qui l'ont vu et entendu ne l'ont pas cru. ${ }^{5}$

\section{3/ Les potentialités ontologiques de la « rétroversion »}

On est néanmoins en droit de penser que la théorie de la résistance à l'information comme preuve de la transcendance de la Parole de Dieu et de son immanence à l'humanité a une portée ontologique radicale dont la phénoménologie de la vie est susceptible de révéler toute la signification sur le plan de la vérité. Cela se vérifie si on considère attentivement la manière dont Tresmontant se rapporte aux Ecritures. Une autre thèse polémique avancée par cet auteur, d'ailleurs liée à celle de la data-

\footnotetext{
${ }^{1}$ C. Tresmontant, Le Christ hébreu, op. cit., p. 250-251.

${ }^{2}$ M. Henry, «Archi-christologie », art. cit., p. 115-116.

${ }^{3}$ C. Tresmontant, Le problème de la révélation, op. cit., p. 144 ; nous soulignons.

${ }^{4}$ M. Henry, «Archi-christologie », art. cit., p. 117.

${ }^{5} \mathrm{M}$. Henry, C'est moi la vérité, op. cit., p. 13.
} 
tion des Ecritures, est celle selon laquelle les Evangiles auraient d'abord été écrits en hébreu - la langue du Christ — pour ensuite être traduits en grec, ce qui constituerait une perte par rapport à la version originale : «Dans le passage de l'hébreu au grec, puis du grec au latin, puis du latin au français, les expressions sont déformées, défigurées, et rendues finalement inintelligibles $»^{1}$. Une nouvelle fois, cette idée ne sera pas acceptée par la critique. Notons ici la réaction de Pierre Grelot, soucieux de préserver l'universalité de la prédication évangélique. Il souligne donc l'absurdité de faire de l'hébreu une langue sacrée et ce en vertu même de la nécessité pour l'enrichissement de l'Evangile d'un contact avec d'autres cultures'.

Ne perdons cependant pas de vue que la démarche de Tresmontant à l'égard du texte biblique récapitule deux acquis de son approche du christianisme qui, une fois rappelés, doivent nous faire comprendre que, dire des évangiles qu'ils sont écrits en hébreu est un propos qui doit moins être lu sur un plan philologique - ce qui serait de fait une absurdité - qu'à un niveau ontologique. D'une part, il est patent que la thèse de Tresmontant fait référence à sa volonté de singulariser la pensée hébraïque dans sa différence avec les schèmes de la pensée grecque. D'autre part, il semble tout aussi évident, que l'idée d'une dégradation du message originaire hébreu dans sa traduction grecque puis dans les autres langues, s'inscrit dans le cadre de l'alternative définissant le destin de la Parole de Dieu comme «information créatrice » dès lors qu'elle se trouve reçue et interprétée par l'humanité. Celle-ci, avons nous déjà évoqué, peut être soit " continuée », soit « dégradée ». Claude Tresmontant, émet la seconde hypothèse au sujet de la traduction grecque des Evangiles. Dès 1969 — bien avant la rédaction du Christ hébreu — il soulignait déjà : « Les traductions de la Bible hébraïque en grec, puis du grec en latin, puis du latin en langue nationale, entraînent une perte d'information $»^{3}$. Or, afin de combler ce qui se présente à lui comme une lacune, il définit à sa manière le rôle et le travail de la critique

\footnotetext{
${ }^{1}$ C. Tresmontant, Le Christ hébreu. op. cit., p. 25, nous soulignons.

${ }^{2}$ «En annonçant l'Evangile dans la langue populaire, Jésus donnait un exemple qui fut certainement suivi aux origines chrétiennes : l'Evangile y fut annoncé dans les langues courantes qu'on parlait : en premier lieu, la langue parlée des disciples galiléens, donc l'araméen; en second lieu, l'hébreu, dans la mesure où il y avait en Judée des gens qui le parlaient; en troisième lieu, le grec, dès que le milieu des Juifs hellénistes put être atteint à Jérusalem même (cf. Ac 6) [...]. Ces faits sont une première ouverture vers l'universalité de la prédication évangélique » (Evangiles et tradition apostolique, op. cit., p. 50-51; nous soulignons). Pour un regard critique sur la manière dont Tresmontant envisage les Ecritures, on renvoie le lecteur à Emilio Rasco, «Deformacion y formacion de los Evangelios. De Claude Tresmontant à Pierre Grelot», dans Gregorianum, 67, 2 (1986), p. 329-339. Certaines recensions permettent également de se faire une idée des immenses problèmes que pose la démarche de Tresmontant : Gerard Sloyan, «Le Christ hébreu », dans Catholic Biblical Quarterly, 47 (1985), p. 745-747 ; J. Winandy, « Le Christ hébreu. La langue et l'âge des Evangiles », dans Nouvelle Revue Théologique, 106 (1984), p. 260.

${ }^{3} \mathrm{C}$. Tresmontant, Le problème de la révélation, op. cit., p. 150 ; nous soulignons.
} 
biblique : « le travail du philologue, de l'exégète, de l'historien, c'est d'essayer de retrouver le texte à sa source $»^{1}$.

Au niveau de la traduction, cette entreprise est menée par le biais de ce que certains auteurs, appartenant à la même mouvance que Tresmontant mais ayant des démarches parallèles, appelleront une « rétroversion $»^{2}$. De la sorte, il convient de retrouver, en-deçà du texte grec, «l'hébreu nu et cru » ${ }^{3}$. Concrètement, souligne l'auteur du Christ hébreu, « il faudra nous demander dans chaque cas quel est le mot hébreu qu'il [le mot grec] recouvre $»^{4}$. Un regard attentif à la manière dont Tresmontant met en œuvre sa démarche, nous fait comprendre que son projet de rétroversion n'a pas une portée philologique. À l'occasion de sa traduction de l'Evangile selon Saint Jean, et spécialement dès lors qu'il envisage le Prologue selon la méthode qu'il préconise, le résultat donne ceci: « c'était la lumière de vérité qui illumine tout homme elle est venue dans le monde de la durée présente. Dans le monde de la durée présente elle était et le monde par sa main a été créé et le monde ne l'a pas connue $»^{5}$ (Jean 1, 9-10). Il est aisé de percevoir que la langue cible, en l'occurrence, le français, censée rendre compte de la richesse de l'hébreu — langue source - est en réalité criblée de concepts philosophiques dont Tresmontant se sert pour singulariser la pensée hébraïque dans sa différence avec la pensée grecque.

L'entreprise qui vise à reconduire les traductions à la « pureté » de l'hébreu a donc un sens ontologique puisqu'on constate que la reformulation de ces quelques versets du Prologue johannique nous renvoie directement au concept de durée, celui-là même qui marquerait la spécificité du monde biblique dont le sens est obtenu à partir des thèses de Bergson. À cet égard, Tresmontant précise, dans le commentaire de Jn 1, 9 qui suit la traduction de ce même passage :

Il ne faut jamais oublier, lorsqu'on lit le quatrième Evangile, que le grec kosmos ne signifie pas ce que signifie en français le monde. En français le monde signifie la même chose que l'Univers physique. Dans le quatrième Evangile kosmos

\footnotetext{
${ }^{1}$ Ibid.

${ }^{2}$ À cet effet, Bernard Dubourg dit bien ceci : «Pour me résumer, je dirai que rien ne s'oppose d'emblée à l'idée d'une rédaction hébraïque primitive de la plupart des textes du Nouveau Testament et, plus particulièrement, des Evangiles. L'hébreu est une langue vivante dans la Palestine du 1er siècle : cette langue se parle, se lit et s'écrit. Par ses aberrations mêmes, le grec des Evangiles tend à se révéler comme de seconde main. De toute ceci, je déduis qu'il faut, au moins à titre d'essai, pratiquer enfin une rétroversion sérieuse et patiente du grec du Nouveau Testament vers l'hébreu ; car n'est-il pas possible, grâce à un examen scrupuleux des manuscrits que nous avons et des versions qu'ils contiennent, mot à mot, phrase après phrase, d'obtenir sinon l'original sémite, du moins une notion plausible de cet original ?" (L'invention de Jésus. I. L'hébreu du Nouveau Testament, Paris, Gallimard, «L'Infini », 1987, p. 20). Si nous faisons allusion à cet ouvrage polémique, c'est parce qu'une étude du manuscrit de C'est moi la vérité, témoigne matériellement du fait que Michel Henry a sinon lu, du moins consulté cette source. On se permet ici de citer la note préparatoire Ms A 32-28-24545 : «B. Dubourg L’invention de Jésus Gallimard ».

${ }^{3}$ C. Tresmontant, Le Christ hébreu. op. cit., p. 28.

${ }^{4}$ Ibid., p. 31.

${ }^{5}$ Evangile de Jean, trad. Tresmontant, op. cit., p. 17 ; nous soulignons.
} 
correspond à l'expression rabbinique olam ha-zeh, la durée présente de ce monde, ou la durée présente de l'histoire. Il ne s'agit pas de l'univers physique. Il s'agit de la durée de l'histoire humaine, de l'humanité en tant qu'elle vit et pense cette durée de l'histoire humaine présente. ${ }^{1}$

Dès 1953, ce thème est présent dans l'Essai puisque, selon l'idée d'une création continue, l'humanité, dans son histoire, est considérée comme une croissance, un développement, c'est-à-dire une maturation intérieure, ce qui témoignerait du fait que «l'humanité est travaillée de l'intérieur et [que] quelque chose est en train de se faire $»^{2}$. Mais il ne s'agit pas pour l'auteur de répéter ce qu'il a par ailleurs déjà montré puisqu'il souligne que ce développement ne se fait pas uniquement dans la douceur, mais que, bien au contraire, il y a, dans la naissance, dans l'aspect de croissance, un "élément négatif, tragique, inhérent à cette genèse $»^{3}$. La philosophie de l'histoire des prophètes d'Israël est en effet marquée par plusieurs aspects qui nous renvoient au concept précédemment évoqué. À cet égard Tresmontant souligne que « la guerre, la dialectique de l'oppresseur et de l'opprimé, la captivité et puis le retour, la libération sont constitutifs de cette durée-ci. 'olam haze $»^{4}$. De la sorte s'éclaire l'interprétation de Jn 1, 9, car si « la lumière de vérité » est venue dans «le monde de la durée présente», alors celle-ci se soumet à cet horizon mouvementé que nous avons déjà évoqué en abordant la problématique de la résistance à l'information. En ce sens, notre thèse est que la communauté entre Claude Tresmontant et Michel Henry se lit non seulement sur le plan de l'authenticité mais également sur le plan de la vérité.

La compréhension du monde comme durée explique que « le monde ne l'a [la lumière véritable] pas connue » ou, comme le suggère avec encore plus de radicalité le onzième verset du Prologue johannique, que « chez ceux qui sont à elle [la lumière de vérité] elle est venue » et que " ceux qui sont à elle ne l'ont pas reçue »" (Jean 1, 11). Les raisons de ce refus évoquent à nouveau la théorie de la " haine du Verbe » mais reprise cette fois sur le plan de la subjectivité. Tresmontant souligne que

le quatrième Evangile propose une théorie et une explication de cette résistance à la lumière : «La lumière luit dans la ténèbre, et la ténèbre ne l'a pas reçue » (Jean 1, 5). «Elle était dans le monde (la lumière), le monde a été fait par elle, et le monde ne l'a pas connue » $(1,9)$. «Elle est venue parmi les siens, et les siens ne l'ont pas reçue » $(1,11)$. « La lumière est venue dans le monde, et les hommes ont aimé davantage l'obscurité que la lumière, car leurs œuvres étaient mauvaises $(3,19){ }^{6}$

\footnotetext{
${ }^{1}$ Ibid., p. 125.

${ }^{2}$ C. Tresmontant, Essai sur la pensée hébraïque, op. cit., p. 80 ; nous soulignons.

${ }^{3}$ Ibid.

${ }^{4}$ Ibid.

${ }^{5}$ Evangile de Jean, trad. C. Tresmontant, op. cit., p. 17.

${ }^{6}$ C. Tresmontant, Le problème de la révélation, op. cit., p. 152 ; nous soulignons.
} 
Or, à cet égard, il est intéressant de constater que Tresmontant assigne l'origine de la résistance à l'information qui, rappelons-le, est un signe de la transcendance et de l'authenticité de la Parole de Dieu, non plus à un contexte historique particulier mais à une modalité du sujet : «On retrouve la notion de résistance [...] dans l'ordre de la psychologie des profondeurs. Freud a appelé "résistance" (Widerstand) "tout ce qui au cours de la cure psychanalytique, dans les actions et les paroles de l'analysé, s'oppose à l'accès de celui-ci à son inconscient" ".. 'Mais ce qu'il importe surtout d'exhiber, c'est la clé herméneutique à partir de laquelle Tresmontant interprète les versets johanniques auxquels nous venons de faire référence :« En somme, la résistance manifeste l'opposition du sujet à ce qu'il prenne conscience de sa propre vérité. Elles attestent le refus acharné, violent souvent, de se voir soi-même tel que l'on est, de mettre la lumière au fond de soi-même ».2

On ne peut attester matériellement le fait que Michel Henry a pu lire les thèses que propose Tresmontant, en ce sens que la seule source effectivement considérée par le phénoménologue se limite au Christ hébreu. Nous sommes pourtant en droit de déceler une certaine influence de la théorie de la résistance à l'information dans l'approche phénoménologique du christianisme. En premier lieu les versets mobilisés par Michel Henry pour esquisser sa théorie de la haine du Verbe, sont précisément Jean 1, 10-11 et Jean 3, 19-2033, c'est-à-dire ceux-là même qu'utilise Tresmontant dans le cadre de sa démarche. En second lieu, sur le plan théorique, Michel Henry se rapproche clairement de Claude Tresmontant puisque l'on retrouve, spécialement dans Paroles du Christ, la thèse selon laquelle la haine, le refus du Verbe, c'est-à-dire la résistance de l'homme à l'égard de la "vraie lumière » s'origine dans le fait que celle-ci est capable, dans sa violence intrinsèque, de le révéler à luimême tel qu'il est. La vérité de la Vie est inscrite au registre de la violence qui est celle «d'une auto-révélation sans retrait ni réserve, sans retard ni discours, que se révélant à soi $[\ldots]$, elle porte immédiatement témoignage sur elle-même » ${ }^{4}$. Et Michel Henry ajoute à cet égard : «c'est dans la Vérité de la Vie absolue, en son autorévélation invincible que chaque moi est révélé en son cœur [...] la révélation à soi de la Vie en chaque Soi vivant habite chacune des modalités de sa vie, de ses joies,

${ }^{3}$ «Parmi les paroles du Christ, il en est qui dénoncent la haine de la vérité, et ce sont les plus terribles [...] le Prologue dit: "Le Verbe était la vraie lumière qui éclaire tout homme venant en ce monde. Il était dans le monde, lui par qui le monde a été fait, mais le monde ne l'a pas reconnu. Il est venu chez les siens, et les siens ne l'ont pas reçu" (versets 10-11). C'est parce que le Verbe est la révélation sans partage qui illumine le secret des cœurs que les hommes voués au mal haïssent le Verbe [...]. Ce que dit le contexte de l'entretien : "Et le jugement, le voici : quand la lumière est venue dans le monde, les hommes ont préféré les ténèbres à la lumière, parce que leurs œuvres étaient mauvaises. En effet tout homme qui fait le mal déteste la lumière" (3, 19-20) » (Paroles du Christ, op. cit., p. 124-125.)

${ }^{4}$ Ibid., p. 123-124.
} 
de ses blessures, des actes qui en résultent, c'est [donc] chacun de ces actes, au moment même où il s'accomplit, qui est connu de Dieu ${ }^{1}$. Or — le problème du mal mis à part — c'est dans le fait d'être systématiquement «démasqué » par «la lumière qui éclaire tout homme » que se fonde le véritable motif de la haine à l'égard du Christ : "À quoi tient cette haine, nous venons de le comprendre. Si chaque modalité de notre vie, chaque mouvement de notre cœur est révélé à soi dans l'autorévélation de la Vie absolue, alors toute pensée mauvaise [...], tout acte qui en résultent se trouvent démasqués $»^{2}$. Dès lors, on comprend que la haine du monde déployée à l'encontre du Verbe atteste son caractère irréductiblement divin dès lors qu'il se déploie dans chaque homme concerné par cette révélation, c'est-à-dire chez les «siens». En guise de conclusion et pour réinstaurer une forme de pudeur, il convient néanmoins de rappeler la remarque nuancée de Jean Grosjean commentant Jean 1, 5 - « le monde ne l'a pas reconnu »: «Il y a des bouderies qui sont le degré d'un amour plus fort, mais il y a celles qui sont les premières failles d'un abîme »'.

${ }^{1}$ Ibid., p. 124.

${ }^{2}$ Ibid. ; nous soulignons.

${ }^{3}$ Jean Grosjean, L'ironie christique. Commentaire de l'Evangile selon Jean, Paris, Gallimard, 1991, p. 23. 
